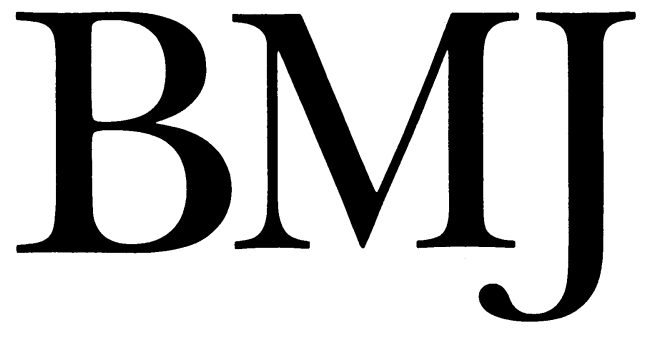

\title{
Zidovudine after occupational exposure to HIV
}

\author{
Hospitals should be able to give it within an hour
}

Health care workers in contact with patients or laboratory specimens are at a low, but real, risk of infection with HIV. There are currently 28 documented case histories of seroconversion in employees who have had no other known risk factors. Studies coordinated by the Centers for Disease Control in the United States have provided an estimate of the risk of infection after substantial occupational exposure: to date, seroconversion has been recorded in six of 1962 participants, who have experienced 2008 percutaneous exposures to HIV positive material.' This indicates a risk of one infection for every 320 exposures $(0 \cdot 31 \%)$. No cases of infection have resulted from 1501 instances of contamination of the mouth or eyes or both, although there have been anecdotal reports of infection from blood splashes to the face.

Much can be done to reduce the risk of exposure. In Britain all employers have a legal obligation under the Health and Safety at Work Act 1974 to ensure that all their employees are properly trained and proficient in safe working practices. In addition, all employees are required by the Control of Substances Hazardous to Health Regulations 1988 to review every procedure that entails contact with a substance hazardous to health, including pathogenic micro-organisms and viruses. Guidelines provide a useful basis from which to prepare detailed local safety documents. ${ }^{2-4}$ Although much may be done to minimise occupational exposure to HIV, accidents will still occur (for some types of surgery the risk of inoculation is high).

Some centres have recommended zidovudine for chemoprophylaxis after exposure. In the body zidovudine's active metabolite interferes with production of HIV DNA, which is controlled by the virus encoded reverse transcriptase. Any activity as a chemoprophylactic agent is likely to occur only after the virus has entered a susceptible cell and before the DNA provirus has been integrated. The early events in HIV infection in humans are poorly understood; on theoretical grounds, and on the basis of cell culture and animal experiments, zidovudine is unlikely to be effective if given after the first cycle of replication of the virus. How efficiently zidovudine inhibits the initial phase of reverse transcription of the virus and blocks replication of HIV in all susceptible cells in vivo is unknown.

Several experimental agents, active against HIV in vitro, have been postulated to prevent the virus entering target cells. These include recombinant soluble CD4 and polyanions such as heparin sulphate and dextran sulphate. These agents have produced no clinical benefit in patients with AIDS, and, although they may seem more suitable for prophylaxis than zidovudine, there is at present no indication for their use. A report of enhancement of simian immunodeficiency virus infection of human cells by soluble CD4 emphasises the need for caution. ${ }^{56}$

Although studies in animals have found that zidovudine may alter the pattern of retroviral infection, there are no reports of prevention of infection. Ruprecht et al showed that zidovudine started four hours after a large inoculation of the Rauscher murine leukaemia virus suppressed viraemia, although this occurred after zidovudine was withdrawn. ${ }^{7}$ Similarly, zidovudine given within one hour prevented viraemia in cats infected with feline leukaemia virus. ${ }^{8}$ One of the cats developed neutralising antibody at 15 weeks, and the investigators postulated control of infection by the development of antibody and cell mediated immunity during a period of virustasis induced by the treatment. There is no evidence that HIV infection can be controlled by the human immune system in a similar way to feline leukaemia virus in cats. Neither of these studies showed prevention of infection as assessed by the polymerase chain reaction or other sensitive techniques.

\section{Primate studies disappointing}

Studies in primates infected with simian immunodeficiency virus failed to show a protective effect of zidovudine even when the drug was given before inoculation with the virus. McClure et al reported the effects of zidovudine given for 14 days, starting one hour, 24 hours, and 72 hours after inoculation of macaques with a small dose of a rapidly lethal variant of simian immunodeficiency virus (HM McClure et al, fifth international conference on AIDS, Montreal, 1989). All the animals treated at 24 and 72 hours became infected, and four out of six died. Two of the three macaques given zidovudine one hour after infection became infected and one died. In another study zidovudine started eight hours before inoculation of macaques with simian immunodeficiency virus failed to prevent viraemia but delayed its onset until one to two days after the completion of treatment (B Lundgren $e t$ al, symposium on non-human primate models for AIDS, San Antonio, Texas, 1988).

Other investigators have used the SCID-hu mouse model, which is prepared by reconstituting the immune system of an immunodeficient mouse with human haematolymphoid 
organs susceptible to HIV. ${ }^{9}$ Seventeen mice were treated with zidovudine for 24 hours before and for two weeks after a standard dose of HIV (400-4000 international units). (This was the smallest dose capable of infecting all the animals by the route used-intrathymic inoculation.) Two weeks after infection none of the mice was positive for HIV DNA by the polymerase chain reaction, although HIV RNA was detected in some cells by in situ hybridisation. Four weeks after zidovudine was stopped HIV DNA was detected by the polymerase chain reaction in all the mice. ${ }^{10}$ The value of animal experiments in assessing the potential protective effect of zidovudine against HIV infection in humans is, however, questionable. The small animal models may be inappropriate as the viruses used are very different from HIV. Furthermore, it is impossible to infect animals with the minute volumes of blood associated with transmission in most cases of occupational exposure, and it may be inappropriate to expect zidovudine to protect against large inoculums of virus given intrathymically or intravenously.

\section{Human studies equivocal}

Can the value of zidovudine given after exposure be assessed from data on human exposures? Although a double blind placebo controlled study of prophylaxis with zidovudine was sponsored by Burroughs Wellcome, enrolment was terminated in June 1989 as it became obvious that the low rate of seroconversion $(0 \cdot 31 \%)$, together with increasing use of the drug after exposure and reluctance to accept the possibility of placebo, would never provide data on efficacy. Of 84 people enroled in the study (49 of whom received zidovudine), none became HIV positive after at least six months of follow up. ${ }^{11}$

There are two published accounts of accidental transmission of HIV in which zidovudine failed to protect the exposed person. Lange et al gave the drug within 45 minutes to a patient who had received an injection with a syringe that had previously been used for a patient infected with HIV.12 The syringe was estimated to contain 100-200 $\mu \mathrm{l}$ of blood. Despite a combination of oral and intravenous zidovudine over more than three months, starting at a dose of $500 \mathrm{mg}$ every six hours, HIV p24 antigenaemia appeared at 30 days and seroconversion occurred at 41 days. A subsequent report of seroconversion after a deep needlestick injury involving blood from a patient with AIDS records failure of prophylaxis with zidovudine, which was started within six hours of the injury. ${ }^{13}$ Discouraging as these reports may seem, no ways of detecting instances of successful prophylaxis exist.

How much toxicity was associated with zidovudine used prophylactically in health care workers? Preliminary data from the placebo controlled study sponsored by Burroughs Wellcome in which participants received $200 \mathrm{mg}$ of zidovudine (or placebo) every four hours for six weeks showed that nausea and vomiting were the commonest adverse effects. "No participant stopped the study drug or placebo because of haematological or other serious toxic reactions. Of the 49 recipients of zidovudine, however, 14 had a haemoglobin concentration between 95 and $120 \mathrm{~g} / \mathrm{l}$ compared with one of 35 patients who received placebo. Seven of the zidovudine group compared with one of the placebo group elected to stop treatment because of subjective, reversible symptoms including nausea, vomiting, fatigue, headache, myalgia, and cough. The risk of long term toxicity, including teratogenic and carcinogenic effects, is unknown. In studies conducted by Burroughs Wellcome, vaginal tumours were observed in mice and rats receiving zidovudine. ${ }^{11}$ In mice these tumours occurred at doses resulting in plasma concentrations similar to those produced by the doses originally approved for treatment of people with AIDS ( $200 \mathrm{mg}$ every four hours). The plasma concentrations associated with tumours in rats were 10 times higher than those resulting from treatment of humans. There are no reliable data on the safety of zidovudine in pregnancy or during breast feeding.

The lack of data on the efficacy and long term safety of using zidovudine prophylactically makes it all too easy to say that zidovudine should not be made available for prophylaxis after exposure. But, in the face of a small but real risk of infection from inoculation injury and the knowledge that there is nothing else to offer, depriving health care workers of any possible benefit from the drug would surely be wrong. Many are asking about its availability after accidental inoculation, and the need for a policy on prophylaxis after exposure is being considered in hospitals, research institutes, and pharmaceutical companies. Many have decided to follow the recommendations introduced at San Francisco General Hospital $^{14}$ and have arranged for zidovudine to be rapidly available in certain defined circumstances when high level exposure to HIV has occurred. Percutaneous inoculation with concentrated preparations of the virus or HIV culture material, transfusion or injection of blood infected with HIV, and lacerations or intramuscular needlestick injuries with blood from HIV positive patients are examples of exposures likely to result in an appreciable risk of percutaneous transmission. Less severe exposure, including finger pulp injuries and splashing of blood into the face, may be considered less risky. Drawing the dividing line may, however, be misleading as factors other than the amount of virus inoculum may be important in transmission-for example, some of the seroconversions reported seem to have resulted from trivial injuries.

On theoretical grounds and with perhaps some support from animal experiments, starting prophylaxis as soon as possible, preferably within an hour of exposure, seems sensible. Several British laboratories working with HIV cultures have arranged for starter doses of zidovudine to be held on site in case of accidental exposure. Arranging for the drug to be available to health care workers in general is much less easy, and administration must be accompanied by the counselling necessary to allow people to decide whether they should accept chemoprophylaxis. During the working week this is probably best arranged through occupational health departments in hospitals, but provision will be needed to cover injuries during the night and at weekends.

\section{Retain records}

Prophylaxis with zidovudine should be followed by regular testing for HIV antibodies and clinical and haematological assessment to monitor possible toxicity. Because any long term effects are unknown records should be retained for retrospective analysis. As zidovudine is likely to be reserved for health care workers who have been exposed to a substantial risk of infection with HIV, advising the recipients to ensure that they reduce the risks of transmission of virus to others is logical. This would include avoiding donating blood or semen and avoiding sexual transmission. A negative result of an HIV antibody test six months after exposure should be sufficient evidence to allow these restrictions to be relaxed.

Professor of Virology,

D J JEFFRIES

St Bartholomew's Hospital,

London EC1A 7BE

1 Marcus R and the CDC Cooperative Needlestick Surveillance Group. Surveillance of health carc workers exposed to blood from patients infected with the human immunodeficiency virus. N Engl F Med 1988;319:1118-23.

2 UK Health Departments. Guidance for clinical health care workers: protection against infection with $H I V$ and hepatitis viruses. Recommendations of the expert advisory group on AIDS. London: HMSO, 1990 
3 Advisory Committee on Dangerous Pathogens. HIV-the causative agent of AIDS and related conditions. Second revision of guidelines. London: Department of Health, 1990

Centers for Disease Control. Guidelines for prevention of transmission of human immunodeficiency virus and hepatitis $\mathrm{B}$ virus to health-care and public-safety workers. MMWR 1989;56:1-37.

Werner A, Winskowsky $G$, Knuth R. Soluble CD4 enhances simian immunodeficiency virus SIVagm infection. I Virol 1990;64:6252-6.

Allan JS, Strauss J, Buck DW. Enhancement of SIV infection with soluble receptor molecules. Science 1990;247:1084-8.

Ruprecht RM, O'Brien LG, Rossoni L.D, Nusinoff-Lehrman S. Suppression of mouse viraemia and retroviral disease by 3'-azido-3'-deoxy thymidine. Nature 1986;323:467-9.

Tavares L Roneker C, Johnston K, Nusinoff-Lehrman S, de Noronka F 3'-azido-3'deoxythyTidine fine leuk Cancer Res 1987:47:3190-4.
9 Namikawa R, Kanishima H, Leiberman M, Weisman IL, McCune JM. Infection of the SCID-hu mouse by HIV-1. Science 1988;242:1684-6.

10 McCune JM, Namikawa R, Shih CC, Rabin L, Kanishima H. 3'-azido-3'deoxythymidine suppresses HIV infection in SCID-hu mouse. Science 1990;247:546-6.

11 Centers for Disease Control. Public health service statement on management of occupationa exposure to human immunodeficiency virus, including considerations regarding zidovudin post-exposure use. $M M W R$ 1990;39:1-14.

12 Lange JMA, Boucher CAB, Hollak CEM, et al. Failure of zidovudine prophylaxis after accidental exposure to HIV-1. N Engl f Med 1990;322:1375-7.

Looke DFM, Grove DI. Failed prophylactic zidovudine after needlestick injury. Lancel 1990;335:1280.

14 Henderson DK, Gerberding JL. Prophylactic zidovudine after occupational exposure to the human immunodeficiency virus: an interim analysis. 7 Infect Dis 1989;160:321-7.

\section{Completeness of reporting of AIDS cases}

\section{Doctors should beware of "reporting fatigue"}

In the United Kingdom the surveillance of AIDS is based on a voluntary confidential reporting scheme. For each confirmed or suspected case doctors are asked to complete a special clinical report, which is then forwarded to the Public Health Laboratory Service Communicable Disease Surveillance Centre (CDSC) in England and Wales or to the Communicable Diseases (Scotland) Unit. For purposes of surveillance cases of AIDS are defined by the presence of specified indicator diseases in people who usually have laboratory evidence of infection with HIV-I.' ${ }^{\prime}$ Since mid-1989 doctors have also been asked to complete clinical report forms on deaths in people known to have been infected with HIV-I but in whom no indicator disease was diagnosed. As an extra safeguard for preserving the confidentiality of a patient's surname reporting doctors are encouraged to use the Soundex alphanumeric code as an alternative. ${ }^{\star}$

These reports provide an insight into the pattern, extent, and trend of the HIV-I epidemic and give information necessary for providing services ${ }^{23}$ and evaluating prevention programmes. Reports on deaths of people infected with HIV-I but without AIDS allow better estimates of the impact of the HIV-I epidemic and contribute to the evaluation of the case definition for AIDS. ${ }^{+}$Doctors appreciate the need for the scheme: by the end of April over 750 doctors throughout the United Kingdom had reported 4568 cases of AIDS.

Evidence exists, however, that the reporting of AIDS is incomplete. ${ }^{5-8}$ As making AIDS a statutorily notifiable disease would not necessarily lead to complete reporting ${ }^{9}$ what other strategies are available for improving reporting?

Supplementary ascertainment of cases is one such method. Possible cases of AIDS are ascertained from death registration data received at the Office of Population Censuses and Surveys and by the registrar general for Scotland; laboratory reports of opportunistic infections; clinical information in laboratory reports of HIV-I infections; reports by directors of haemophilia centres to the secretariat at the Oxford Haemophilia Centre; and regular returns by paediatricians to the British Paediatric Surveillance Unit. ${ }^{+}$Doctors of patients identified by these methods are invited to complete a clinical report.

Comparing the number of cases of AIDS obtained by supplementary ascertainment with the number that has been reported to national registers gives an estimate of underreporting, although interpreting the difference is difficult for several reasons. Firstly, cases of AIDS are frequently ascertained through multiple channels and are

^Copies of the current case definition, clinical report forms, Soundex code guide, and guidance on reporting are available from the CDSC (081 2006868 ) and Communicable Diseases (Scotland) Unit (041 946 7120) and from local consultants in communicable diseases, genitourinary medicine, infectious diseases, and microbiology. usually reported eventually; thus the boundary between validation of surveillance and surveillance itself is blurred. Secondly, for any condition of long duration with an increasing incidence a delay between diagnosis and reporting will be inevitable and underreporting constant. For example, of the 358 cases of AIDS reported during the first three months of 1991, 30 had been diagnosed in 1989, 15 in 1988, and six in 1987 or earlier. (Mathematical techniques can adjust for this delay..$^{10}$ )

Thirdly, some methods of ascertaining suspected casessuch as the use of death entries - are relatively quick, and this should be allowed for when comparisons are made. Fourthly, unreported "possible cases of AIDS" are a heterogeneous group comprising diagnosed cases, unrecognised cases, severe disease related to HIV-I infection not meeting the criteria for AIDS, coincidental diseases unrelated to HIV-I infection, and diseases in people not infected with HIV-I.

The CDSC has adopted several approaches to estimate underreporting. Since 1989,1414 possible cases of AIDS from 20 districts have been sought in the national register: $1102(78 \%)$ had been reported. A further $113(8 \%)$ were later reported, and, given that many of the remaining subjects had yet to develop AIDS, the underreporting rate for established cases of AIDS in these districts would be less than $14 \%$. Last year extensive case searching in three districts found that only $28(4 \%)$ of 666 cases of AIDS diagnosed locally in 1988 or earlier had not been reported to the CDSC. Of the patients with AIDS who had received respite and terminal care at two London centres, about one in 10 had not previously been reported. At the time of a study of laboratory reports of selected opportunistic infections in people who were also infected with HIV-I about $10-15 \%$ had not been reported as having AIDS.

In this issue McCormick has compared estimated excess deaths from 86 medical causes possibly related to HIV-I infection with the number of reported deaths from AIDS and concluded that $92 \%$ of deaths from AIDS in 1989 were reported (p 1375). ${ }^{11}$ As more deaths from AIDS in 1989 will probably be reported in 1991 and 1992 the underreporting rate will fall further. Current data therefore suggest that the rate of underreporting of cases of AIDS in the United Kingdom lies between $5 \%$ and $20 \%$.

The most effective way of ascertaining cases locally is to review the clinical state of people known to be infected with HIV. As Hickman and colleagues show, the number of people known to be infected with HIV may be substantially greater than the number reported nationally ( $p$ 1376). ${ }^{12}$ Although the authors recognise that the size of the discrepancy may be unique to Riverside district, their results emphasise the value of audit.

Other work reported in this issue shows the need for 